\title{
Assessing the relative impact of lupus nephritis on mortality in a population-based systemic lupus erythematosus cohort
}

\author{
SE Reppe Moe ${ }^{1}, \varnothing$ Molberg ${ }^{1,2}$, EH Strøm ${ }^{3}$ and K Lerang ${ }^{1}$ \\ ${ }^{1}$ Department of Rheumatology, Oslo University Hospital, Oslo, Norway; ${ }^{2}$ Institute of Clinical Medicine, University of Oslo, Oslo, Norway; and \\ ${ }^{3}$ Department of Pathology, Oslo University Hospital, Oslo, Norway
}

\begin{abstract}
Objective: There is limited knowledge on the relative impact of lupus nephritis (LN) on morbidity and mortality in population-based systemic lupus erythematous (SLE) cohorts. Here, the primary aim was to compare mortality rates between patients with and without $\mathrm{LN}$ in a population-based SLE cohort. Methods: The study cohort included all SLE patients resident in the city of Oslo during 1999-2008. Follow-up time was median 14 (0-15) years. Presence of LN was defined according to the 1987 American College of Rheumatology classification criteria for SLE. LN class was determined by renal biopsy. Data on kidney function, including presence of end-stage renal disease (ESRD), were obtained from patient charts. Standardized mortality ratios (SMRs) were estimated by comparing deaths in the SLE cohort with age- and gendermatched population controls. Results: We found that 98/325 SLE patients (30\%) developed LN, $92 \%$ of whom had occurrence within the first five years from disease onset. Incidence rate of ESRD was 2.3 per 1000 patient-years. A total of 56 deaths occurred during the study period, corresponding to an overall SMR in the SLE cohort of 2.1 (95\% confidence interval (CI) $1.2-$ 3.4). Estimated SMR for LN patients was 3.8 (95\% CI 2.1-6.2), and for SLE patients without LN it was 1.7 (95\% CI 0.9-2.7). Conclusion: In this population-based SLE cohort, we found that LN was associated with increased morbidity and mortality, whereas SLE patients who did not develop LN had good overall prognoses regarding survival. Lupus (2019) 28, 818-825.
\end{abstract}

Key words: Epidemiology; lupus nephritis; mortality; outcome; systemic lupus erythematosus

\section{Introduction}

Systemic lupus erythematosus (SLE) is an autoimmune, relapsing-remitting, multiorgan disease with a female predominance. Estimates on the incidence and population prevalence of SLE range widely across populations. ${ }^{1-3}$ Based on data from our population-based Oslo County SLE cohort, we recently estimated the incidence of SLE in Norway to be 3.0 per 100,000 per year $(95 \%$ confidence interval (CI) 2.4-3.5). ${ }^{4}$

Renal involvement appears to be common in SLE, with immune-mediated glomerulonephritis (i.e. lupus nephritis; LN), which may be accompa- nied by tubulointerstitial or vascular lesions, as the major disease entity. $\mathrm{LN}$ is a heterogeneous and

Correspondence to: Sigrid Elise Reppe Moe, Department of Rheumatology, Oslo University Hospital, Rikshospitalet, Postboks 4950 Nydalen, 0424 Oslo, Norway.

Email: sigrid@ repmoe.no difficult-to-treat disease. According to recent American and European guidelines, LN is defined as a combination of clinical and laboratory manifestations and is confirmed by renal biopsy. ${ }^{5,6} \mathrm{LN}$ is classified into six different, but partly overlapping classes by validated histopathological criteria from the World Health Organization (WHO). ${ }^{7}$ Existing data indicate large differences in $\mathrm{LN}$ prevalence among SLE populations, with reported frequencies of $\mathrm{LN}$ ranging from $12 \%$ to $69 \% .^{8}$ The frequency and severity of LN appears higher in non-European and younger patients. ${ }^{8,9}$

Even though overall prognosis has improved significantly from the mid-20th century, SLE is still associated with increased mortality. ${ }^{10-12}$ It appears that the presence of $\mathrm{LN}$ and end-stage renal disease (ESRD) per se are important predictors of death. ${ }^{13}$ Studies from tertiary referral centres have reported up to a nine-fold risk of death conferred by $\mathrm{LN},{ }^{14}$ but to the best of our knowledge, there are no population-based estimates on LN-related mortality. 
In Norway every citizen has equal access to health care through the national, state-financed healthcare system. Patients with complex inflam- matory rheumatic diseases, such as SLE, are predominantly managed long term by specialists at the public hospitals, providing a unique opportunity to gather a complete SLE cohort capturing the whole disease spectrum and investigate long-term outcome.

In the Oslo County SLE cohort, we retrieved data from all SLE patients alive and living in Oslo from 1999 through 2008 through compre- hensive searches across multiple sources. ${ }^{4}$ In the current study, we provide long-term follow-up data on LN and its impact on outcome in the Oslo County SLE cohort, and we compare the overall clinical characteristics of patients with and without LN.

\section{Patients and methods}

\section{Study area}

This study was conducted in Oslo, which is the largest city in Norway, with a population of 647,676 inhabitants (1 January 2015). Approximately $20 \%$ of the inhabitants are of non-European origin. Most specialists are hospital based, with only two rheumatologists in private practice in Oslo at the time of the study.

\section{Study cohort}

The Oslo SLE cohort has been described in detail previously. ${ }^{4}$ Briefly, it included all adult (age 16 years) SLE patients alive and living in the study area Oslo in the period of 1 January 1999 to 1 January 2008. Multiple sources were used to secure that all patient information was captured, including in- and outpatient hospital diagnosis registries from all six hospitals in Oslo, The Norwegian Systemic Connective Tissue Disease and Vasculitis Registry (NOSVAR, a local cohort from 1995), ${ }^{15}$ private rheumatologists and Norway's Cause of Death Registry. All patients included met the updated 1997 American College of Rheumatology (ACR) classification criteria for SLE. ${ }^{16}$ This cohort consisted of 325 SLE patients, of whom 131 were incident cases. We collected information on year of diagnosis (as reviewed by a physician), ethnicity (defined as parents' coun- try of birth), treatment received and antibody profiles.

\section{Controls}

For the standardized mortality rate (SMR) ana- lyses, we compared each patient against five con- trols drawn from the national population registry. Patients and controls were matched for place of residency (Oslo), age, gender and parents' ethnicity and vital status at start of follow-up. Dates of death from 1 January 1999-1 January 2014 were retrieved from Statistics Norway. ${ }^{17}$

\section{Follow-up time}

This is a longitudinal, observational, populationbased cohort study. Follow-up started at the time when the patient was registered with an International Statistical Classification of Diseases, revision 10, code of SLE and ended at death, emi- gration or end of study period. Exposure to differ- ent immunosuppressive treatment was recorded until 1 January 2008. Six patients were lost to follow-up because of emigration out of Norway.

\section{Longitudinal assessment of $L N$}

LN was defined by the ACR criteria (proteinuria $>0.5 \mathrm{~g} / 24$ hour and/or relevant casts in urine sediment) for $\mathrm{LN} .{ }^{16}$ A patient who fulfilled the ACR criteria for $\mathrm{LN}$ but did not have a representative renal biopsy available was defined as clinical LN. LN class III, IV or mixed classes with III/V or IV/V were defined as a proliferative type of LN.

At onset of nephritis we recorded kidney function

(reduced or not), hypertension (defined as systolic blood pressure c $140 \mathrm{mmHg}$ or diastolic c $90 \mathrm{mmHg}$ ), presence of nephrotic syndrome

(proteinuria $3.0 \mathrm{~g} / 24$ hour, hypoalbuminaemia of $<2.5 \mathrm{~g} / \mathrm{dl}$ and oedema) and nephritic sediment (urine cellular casts or dysmorphic urinary red cells). For the renal outcome analyses, we recorded data on renal status at last follow-up (the last medical visit before 1 January 2015). Reduced renal function was defined as glomerular filtration rate less than 60 $\mathrm{ml} / \mathrm{min}$ per $1.73 \mathrm{~m}^{2}$ or s-creatinine $>100 \mathrm{mmol} / \mathrm{l}$ for men and $>90 \mathrm{mmol} / \mathrm{l}$ for women and proteinuria as urine albumin to creatinine ratio $>30$ in spot urine. ESRD was defined as the need for chronic dialysis treatment or renal transplantation.

\section{Renal biopsies}

Renal biopsies were retrieved from The Norwegian Renal Biopsy Register and local hospital register. In patients with multiple renal biopsies, only the first biopsy was included. The histological 
classification of $\mathrm{LN}$ was recorded according to the modified WHO criteria. ${ }^{7}$ Five renal biopsies performed before 1990, which were not classified according to these criteria, were re-evaluated by an experienced pathologist (EHS) in 2015 and classified by WHO criteria.

\section{Statistical analysis}

Categorical data were analysed by cross tables and chi-squared test. To estimate risk of death in LN patients, we performed calculations of SMR. Prevalent and incident cases alike were included. SMR, stratified according to age, was calculated by dividing the number of deaths by the number of years observed in every subgroup. The expected number of deaths referred to the number of deaths for the matched control group within the same period of time. The $95 \%$ CI of SMR was calculated using the exact method. ${ }^{18}$ Cumulative incidence of LN was determined by the Kaplan-Meier method, in which time zero was the time SLE was diag- nosed. Allcause ESRD incidence rates were calcu- lated as the total number of patients initiating ESRD treatment during follow-up, divided by the sum of all patientcontributed follow-up years. The calculations were made in SPSS 22. Level of signifi- cance was set at $p$ $<0.05$ in all analyses.

\section{Ethics}

The Regional Committee for Medical Research Ethics and National Data Inspectorate approved this study (nr 2009/2577/REK sør-øst D).
Results

\section{Development of $L N$}

The Oslo SLE cohort included all SLE patients resident in Oslo between 1999 and 2008. Disease characteristics and antibody profiles of the 325 patients in the cohort are summarized in Table 1. During a median follow-up time of 14 years (0-15), clinical LN developed in 98 of the 325 SLE patients from the Oslo cohort. Seventy-four $(76 \%)$ of the 98 patients with LN had the diagnosis confirmed by renal biopsy (Figure 1).

We found that the accumulated frequency of $\mathrm{LN}$ differed between cohort patients of European and non-European descent. The SLE patients of nonEuropean descent were predominantly Asian ( $n$ $34 y_{4}$ The others were African ( $n$ 6) dind South American ( $n$ 5), whereas seven were for- eignadopted, mainly from Asia.

The cohort of patients with LN fulfilled more ACR criteria (mean value 6.0 vs 5.3, $p$ 0.(19)0) and had a higher incidence of haematologic manifestations with thrombocytopaenia (29\% vs $16 \%$, $p^{1 / 4} 0.0092$ ), leucopenia (56\% vs $43 \%, p^{1 / 40.042)}$ and haemolytic anaemia (10\% vs $4 \%, p$ 01@172), as well as malar rash (57\% vs $44 \%$, $p^{1 / 40.002) . ~ I n ~}$ contrast, the non-LN patients had a higher frequency of discoid rash (13\% vs $8 \%, p$ 0.127) and were more likely to have anti-SSA and/or SSB antibodies as a sole antinuclear antibodies subset (8\% vs $2 \%, p^{1 / 4} 0.043$ ). No other clinical

Table 1 Characteristics of the total Oslo SLE cohort, and the patient subsets with and without LN

\begin{tabular}{|c|c|c|c|c|}
\hline & $\begin{array}{l}\text { All } \\
\text { N 1/4 } 325\end{array}$ & $\begin{array}{l}L N \\
\mathrm{~N}^{1 / 4} 998\end{array}$ & $\begin{array}{l}\text { Non-LN } \\
\mathrm{N} 1 / 4227\end{array}$ & $\mathrm{p}$ value $^{a}$ \\
\hline Of non-European descent, $n(\%)$ & $52(16)$ & $26(26)$ & $26(11)$ & 0.001 \\
\hline Age at diagnosis, median (range) & $32(7-83)$ & $22(7-82)$ & $37(10-83)$ & $<0.001$ \\
\hline Age at onset, median (range) & $28(7-82)$ & $21(7-82)$ & $32(9-71)$ & $<0.001$ \\
\hline Age at study end ${ }^{b}$, median (range) & $52(20-99)$ & $42(20-89)$ & $58(22-99)$ & $<0.001$ \\
\hline Disease duration $^{\mathrm{c}}$, median (range) & $16(1-49)$ & $19(1-47)$ & $15(2-49)$ & NS \\
\hline \multicolumn{5}{|l|}{ Antibody profile } \\
\hline ANA, $n(\%)$ & $323(99)$ & $97(99)$ & $226(100)$ & NS \\
\hline Anti-dsDNA, $n(\%)$ & $206(63)$ & $79(81)$ & $127(56)$ & $<0.001$ \\
\hline Anti-Sm, $n(\%)$ & $48(15)$ & $24(24)$ & $24(11)$ & 0.027 \\
\hline Anticardiolipin, $n(\%)$ & $96(30)$ & $23(23)$ & $73(32)$ & NS \\
\hline aPL, $n(\%)$ & $127(39)$ & $39(40)$ & $88(39)$ & NS \\
\hline Anti-SSA, $n(\%)$ & $135(42)$ & $32(33)$ & $103(45)$ & 0.051 \\
\hline Anti-SSB, $n(\%)$ & $60(18)$ & $10(10)$ & $50(22)$ & 0.006 \\
\hline
\end{tabular}

ANA: antinuclear antibodies; Anti-dsDNA: anti-double-stranded DNA; aPL: antiphospholipid antibodies; LN: lupus nephritis, defined after American College of Rheumatology criteria; NS: nonsignificant; SLE: systemic lupus erythematosus.

${ }^{a}$ For the difference between the two subgroups, LN and non-LN patients.

${ }^{\mathrm{b}}$ Age at study end, death or emigration.

${ }^{\mathrm{c}}$ Time from diagnosis to study end, death or emigration. 
manifestation showed a statistically significant difference between the two groups.

Kaplan-Meier analyses of the incident SLE cases showed that $17 / 31$ patients $(55 \%)$ who developed LN did so within the first year, 24/31 (77\%) within two years and 29/31 (94\%) within five years after SLE diagnosis. When analysing the total SLE cohort (incident and prevalent cases), 90/98 (92\%) cases presented with LN within the first five years after diagnosis. However, presentation of renal involvement was seen up to 17 years after diagnosis.

Patients with $\mathrm{LN}$ had received more aggressive treatment than the non-LN patients, with

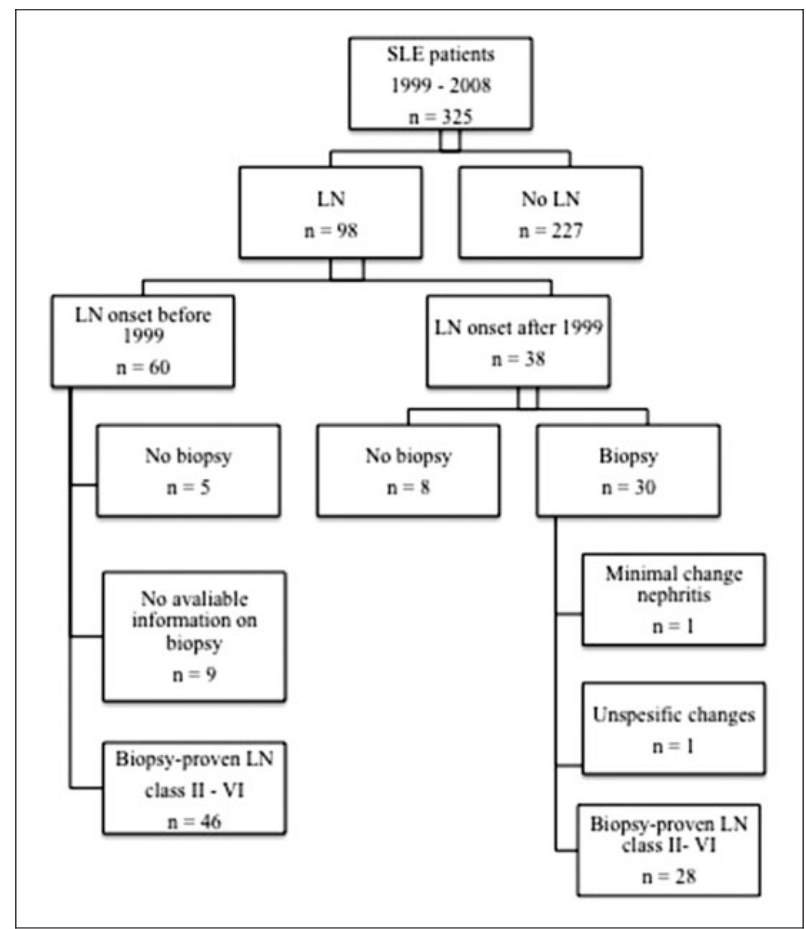

Figure 1 Flow diagram for inclusion of lupus nephritis (LN) in the study.

SLE: systemic lupus erythematosus; LN: lupus nephritis.. cyclophosphamide $(53 \%$ vs $4 \%, p<0.001)$, azathioprine $(66 \%$ vs $27 \%, p<0.001$ and highdose steroids ( $81 \%$ vs $22 \%, p$ 0 000$)$ ). There was no difference in use of antimalarial medication during disease course between the two groups (71\% vs $81 \%, p$ nonsignificant). However, the non-LN patients had more often received antimal- arial medication within the same year of diagnosis compared with those who did develop LN (68\% vs $51 \%, p^{1 / 40.020)}$.

\section{LN class distribution}

Scoring of LN class according to the WHO classification system was performed for the 74 patients who had renal biopsies available for assessment. Two (3\%) patients showed a mixed histologic pattern, class VbIII. In 22 patients (22\%), no renal biopsy was performed, or there was no available information on whether biopsy was performed (Figure 1). In one patient the renal biopsy was described as minimal change disease, and one patient had unspecific minor changes; these two patients were thus defined within the clinical LN group.

Clinical findings at the time of LN diagnosis appeared to differ by LN class (Table 2), exemplified by differences between class III and IV regarding nephrotic syndrome $(19 \%$ vs $81 \%, p<0.001)$ and hypertension $(56 \%$ vs $75 \%, p \quad 0.024)$ (Table 2 ). For further analysis we grouped the 54 patients having class III, IV or mixed class III/IV together as proliferative LN. In three of these 54 patients $(6 \%)$, the only signs of renal disease were nephritic sediment and low-grade proteinuria.

\section{Long-term renal outcome}

During a mean 18.4 years of follow-up (Table 3), $20 / 325$ patients $(6 \%)$ in the total cohort developed ESRD. The majority of the ESRD cases were women; their median age at diagnosis was 19

Table 2 Clinical presentation of LN at the time of diagnosis

\begin{tabular}{|c|c|c|c|c|c|c|c|c|}
\hline & & Biops) & edcases & $\operatorname{lass}\left(\mathrm{n}^{1 / 4}\right.$ & & & & \\
\hline & $\begin{array}{l}\text { All } L N \\
\mathrm{~N}^{1 / 498}\end{array}$ & $\begin{array}{l}I I \\
\mathrm{~N}^{1 / 412}\end{array}$ & 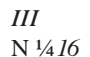 & $\begin{array}{l}I V \\
\mathrm{~N}^{1 / 436}\end{array}$ & $\begin{array}{l}V \\
\mathrm{~N}^{1 / 46}\end{array}$ & $\begin{array}{l}V I \\
\mathrm{~N}^{1 / 42}\end{array}$ & $\begin{array}{l}I I I \mathrm{p} V \\
\mathrm{~N}^{1 / 4} 2\end{array}$ & $\begin{array}{l}\text { Clinical } L N \\
\mathrm{~N}^{1 / 4} 24\end{array}$ \\
\hline Nephritic sediment, $n(\%)$ & $60(61)$ & $8(67)$ & $11(69)$ & $25(70)$ & $1(17)$ & 0 & $2(100)$ & $13(54)$ \\
\hline Hypertension, $n(\%)$ & $47(48)$ & $3(25)$ & $9(56)$ & $27(75)$ & $1(17)$ & $1(50)$ & 0 & $6(25)$ \\
\hline Nephrotic syndrome, $n(\%)$ & $47(48)$ & $1(8)$ & $3(19)$ & $29(81)$ & $3(50)$ & $1(50)$ & $1(50)$ & $9(38)$ \\
\hline Reduced renal function, $n(\%)$ & $48(49)$ & $2(17)$ & $10(67)$ & $22(61)$ & $1(17)$ & $2(100)$ & $1(50$ & $10(42)$ \\
\hline
\end{tabular}

Clinical LN: defined clinically after American College of Rheumatology criteria with no biopsy; hypertension: systolic blood pressure c $140 \mathrm{mmHg}$ or diastolic c $90 \mathrm{mmHg}$; LN: lupus nephritis; nephritic sediment: urine cellular casts or dysmorphic urinary red cells; nephrotic syndrome: proteinuria $3.0 \mathrm{~g} / 24$ hours, hypoalbuminemia of $<2.5 \mathrm{~g} / \mathrm{dl}$ and oedema; reduced renal function: estimated glomerular filtration rate $<60$ or s-creatinine $>100 \mathrm{mg}$ $\mathrm{mol} / \mathrm{l}$ for $\mathrm{men}$ and $>90 \mathrm{mg} \mathrm{mol} / \mathrm{l}$ for women. 
Table 3 Renal outcomes in the LN cohort (at last patient follow-up)

\begin{tabular}{lcccc}
\hline & \multicolumn{4}{c}{ Renal outcome, $\mathrm{n}(\%)$} \\
\cline { 3 - 5 } & $\mathrm{N}$ & Proteinuria $^{a}$ & $\begin{array}{c}\text { Reduced renal } \\
\text { function }^{b}\end{array}$ & ESRD \\
\hline All LN & 98 & $24(24)$ & $49(50)$ & $20(20)$ \\
Class II & 12 & $1(8)$ & $4(33)$ & 0 \\
Class III & 16 & $1(6)$ & $8(50)$ & $3(19)$ \\
Class IV & 36 & $16(44)$ & $22(61)$ & $11(30)$ \\
Class V & 6 & $2(33)$ & $3(50)$ & $1(17)$ \\
Class VI & 2 & 0 & $2(100)$ & $2(100)$ \\
Class III p V & 2 & $1(50)$ & $1(50)$ & 0 \\
Clinical LN & 24 & $3(13)$ & $9(38)$ & $3(13)$ \\
\hline
\end{tabular}

ESRD: end-stage renal disease; LN: lupus nephritis.

${ }^{a}$ Urine albumin to creatinine ratio $>30$ in spot urine.

${ }^{\mathrm{b}}$ Estimated glomerular filtration rate $>60$ or s-creatinine $<100 \mathrm{mg} \mathrm{mol} / 1$

for men and $<90 \mathrm{mg} \mathrm{mol} / \mathrm{l}$ for women.

${ }^{\mathrm{c} C}$ Clinical LN: defined clinically after American College of

Rheumatology criteria, no biopsy.

(7-53) years, and the median time from diagnosis to ESRD was 11 years.

Separate analyses of the 31 incident $\mathrm{LN}$ cases in the cohort showed that only 3/31 developed ESRD during a mean 10.6 years of follow-up, corresponding to an estimated ESRD incidence of 2.3 (95\% CI $-0.3,4.9)$ per 1000 patient-years among newly diagnosed SLE patients.

Detailed chart review on all the $80 \mathrm{LN}$ patients in the total Oslo SLE cohort who were alive at the end of the follow-up period showed that 49/80 $(61 \%)$ had reduced renal function and/or persistent proteinuria (Table 3 ). In these patients, we found that class III and IV LN differed in occurrence of proteinuria ( $6 \%$ vs $50 \%, p^{1 / 4} 0.006$ ), but not in reduced renal function (50\% vs $\left.61 \%, p^{1 / 4} 0.65\right)$.

\section{Mortality}

By the end of the study observation period (after mean follow-up time of 11 years) there were 56 deaths (46 women and 10 men) in the total SLE cohort. Seventeen of the 56 deceased patients (30\%) were diagnosed with LN and 5/17 (29\%) had ESRD. The median disease duration at time of death was 14 years for the $\mathrm{LN}$ patients compared with 13 years for the non-LN patients, but the median age at death was lower for the LN patients than for the non-LN patients (57 vs 69 years, $p 0.05$ ).

$1 / 4 \mathrm{Ve}$ found that the SMR for the total SLE cohort was 2.1 (CI 1.2-3.4). Subgroup analyses showed that the SMR differed between patients with and without LN (3.8 vs 1.7) (Table 4). The highest SMR
Table 4 Standardized mortality rate (SMR), total and stratified by age

\begin{tabular}{|c|c|c|c|c|c|c|}
\hline \multirow[b]{2}{*}{ Age group } & \multicolumn{3}{|c|}{$L N$ patients } & \multicolumn{3}{|c|}{ Non-LN patients } \\
\hline & Deceased & $S M R$ & $C I$ & Deceased & $S M R$ & $C I$ \\
\hline $16-39$ & 6 & 13.4 & $7.2-22.1$ & 1 & 1.6 & $0.2-5.3$ \\
\hline $40-59$ & 2 & 2.2 & $0.5-5.5$ & 11 & 2.2 & $1.1-3.9$ \\
\hline $60-79$ & 6 & 4.9 & $3.8-6.1$ & 18 & 1.8 & $1.0-2.8$ \\
\hline c80 & 2 & 5.2 & $4.5-6.0$ & 10 & 1.6 & $1.2-2.0$ \\
\hline Total & 16 & 3.8 & $2.1-6.2$ & 40 & 1.7 & $0.9-2.7$ \\
\hline
\end{tabular}

estimates were noted in LN patients between age 16 and 39 years (Table 4). In patients with a proliferative class of LN, the SMR was estimated to 4.4 (2.67.0) and 5.4 (3.3-8.2) in patients with ESRD. Causes of death were not recorded.

\section{Discussion}

In this study, we investigated the impact of LN on all-cause mortality and renal outcome in a population-based setting. The main finding was that $\mathrm{LN}$ accounted for most of the increased mortality seen in SLE. To our knowledge, this is the first study in which SMR has been directly compared across a complete SLE cohort stratified by LN status.

At $30 \%$, the $\mathrm{LN}$ frequency in our cohort was comparable to other population-based SLE cohort studies. ${ }^{1,19-23}$ The significantly higher cumu- lative LN incidence we observed in individuals of nonEuropean descent (mainly Asians) is in line with a report from the United Kingdom, ${ }^{24}$ whereas the difference was not so pronounced in a multiethnic United States (US) cohort from New York. ${ }^{25}$

In our study, LN was often present already at the time of SLE diagnosis, and the vast majority (78\%) developed LN within two years, strengthening the notion that risk of $\mathrm{LN}$ decreases with time from diagnosis. ${ }^{26-28}$ Similar to previous studies, the LN patients were younger at diagnosis, fulfilled more ACR criteria and more often had presence of antidouble-stranded DNA, indicating higher disease activity in this group. ${ }^{26,29-31}$ In our study, LN was also associated with anti-Sm. Previous data on this have been less consistent. ${ }^{29-31}$ We found no differences in sex. The protective effect of discoid lupus in our study corroborates observations from the GLADEL (Grupo Latinoamericano de Estudio del Lupus Eritematoso) cohort. $^{32}$ SLE 
patients without LN had their first symptom of 
SLE around age 32 years, 10 years later than patients who developed LN. Furthermore, the diag- nostic delay was much longer in non-LN patients (five years vs one year), indicating two different subsets of SLE.

We observed more frequent use of corticosteroids and aggressive immunosuppression in the LN patients. This is to be expected and in line with current treatment guidelines. ${ }^{5,6}$ Interestingly, we saw a lower occurrence of $\mathrm{LN}$ in patients who start antimalarial treatment within one year of dis- ease onset (68\% vs 51\%, $p$.0203), indicating a protective effect of antimalarial medication on LN development. Similar figures have been reported from Denmark. ${ }^{30} \mathrm{We}$ did not, however, find any difference in the total use of antimalarial medication between the LN and non-LN groups.

Taken together, our results support that close monitoring of renal symptoms in young SLE patients of both sexes is required, especially within the first two years of diagnosis. We believe that the European League Against Rheumatism guideline recommendation of urine test every six to 12 months for inactive disease might not be suf- ficient for the first two years of disease, particularly not in patients of non-European descent. ${ }^{33}$

In our study, the majority of patients $(55 \%)$ had a proliferative LN (III/IV or mixed class). Proliferative LN was mostly associated with an aggressive presentation, but $6 \%$ of these patients had no other clinical manifestation than nephritic sediment and low-grade proteinuria, demonstrating that subtle clinical features can mask severe kidney involvement, and underlining the importance of renal biopsy for further treatment decisions in LN. 5,6

Overall, we found that $6 \%$ of the total SLE cohort (20/325 patients) progressed to ESRD, indicating that ESRD is not a rare outcome in SLE, even in a country where the health expenditure per capita is among the highest worldwide. ${ }^{34}$ In this study of mostly Caucasian patients, the ESRD incidence rate was 2.3 per 1000 patient-years, significantly lower than previously reported from the US and Taiwan, where the respective rates were 12.5 and 6.1 per 1000 patient-years. ${ }^{35,36}$ However, the ESRD rate (3.3) among white SLE patients in the US study was comparable to what we observed. ${ }^{35}$ As a corollary, the observed incidence rate of ESRD related to SLE was as high as the rate of ESRD related to diabetes type 1 in Norway (1.2 per 1000 patient-years). ${ }^{37}$

We report an overall SMR estimate (2.1) for SLE, which mirrors previous population-based studies in the region, ${ }^{38,39}$ as well as two recent meta-analyses. ${ }^{40,41}$ Our data suggest that LN alone accounts for much of the increased mortality in SLE. SLE patients who did not develop LN had an overall good prognosis with a slightly elevated SMR (1.7), but no significant higher mortality com- pared with the general population. This is excellent news for this patient group. To the best of our knowledge, this study is the first to demonstrate this finding. Importantly, whereas young SLE patients without LN showed no increased mortal- ity, we found a very high SMR in young patients with renal disease (SMR 13.4). We also found that patients with proliferative LN had a high mortality rate (SMR of 4.4) and more often developed ESRD. Our findings add to existing data that pro-

liferative LN may predict a poor outcome in SLE. ${ }^{12,14,42}$

There are many reports on SMR in LN, with estimates ranging from 3.6 in Japan to 9 in Chinese SLE patients with biopsy-proven $\mathrm{LN},{ }^{12,14,42-45}$ but none of these studies were per- formed on an SLE cohort at the population level. Two studies from regional centres have indicated high mortality in Scandinavian SLE patients with biopsy-proven LN (SMR of 5.6 and 6.8). ${ }^{12,42}$ Our somewhat lower SMR estimate might be due to the study design, as well as including not only biopsy- proven LN cases.

Despite recent advances in LN therapy, only $61 \%$ of the LN patients who were alive at the end of follow-up had preserved renal function. As previously shown, LN class VI has the worst prognosis, followed by proliferative LN. ${ }^{12,27}$ Additionally, we found that class IV patients presented with more symptoms and had slightly worse prognosis than class III.

The strength of this study is the comprehensive search and identification of SLE patients to minimize the effect of selection bias. Through a comprehensive search for biopsies and the re-evaluation of old biopsies, we found a relatively high number of individuals with biopsy-proven LN $(76 \%)$ compared with a larger international cohort. ${ }^{26}$ However, the results should be read with some cau- tion because of the relatively low number of patients in the cohort. Another limitation is that we did not have access to causes of death or treat- ment received for the whole observational period.

In summary, we report that most LN develops within the first two years of disease. Patients who develop LN have an almost four-fold higher risk of death compared with healthy controls and a 13-fold higher risk in the younger age groups. This warrants a prompt follow-up of LN patients, especially from a young age and for those with class IV LN. 
However, SLE patients without renal involvement have a good overall prognosis.

\section{Acknowledgements}

We would like to thank Rune Bjørneklett, former head of the Norwegian Kidney Biopsy Registry, for his help in gathering renal information about the patients, as well as Gudrun Norby, Department of Rheumatology, Oslo University Hospital, for helpful advice.

\section{Declaration of conflicting interests}

The authors declared no potential conflicts of interest with respect to the research, authorship, and/or publication of this article.

\section{Funding}

The authors disclosed receipt of the following financial support for the research, authorship, and/or publication of this article: This work was supported by the Norwegian Resource Centre for Women's Health and by Grants from the Oslo Sanitetsforenings Legacy and Vivi Irene Hansens Legacy.

\section{References}

1 Somers EC, Marder W, Cagnoli P, et al. Population-based incidence and prevalence of systemic lupus erythematosus: The Michigan Lupus Epidemiology and Surveillance program. Arthritis Rheumatol 2014; 66: 369-378.

$2 \mathrm{Yu} \mathrm{KH}$, See LC, Kuo CF, Chou IJ, Chou MJ. Prevalence and incidence in patients with autoimmune rheumatic diseases: A nationwide population-based study in Taiwan. Arthritis Care Res (Hoboken) 2013; 65: 244-250.

3 Hermansen ML, Lindhardsen J, Torp-Pedersen C, Faurschou M, Jacobsen S. Incidence of systemic lupus erythematosus and lupus nephritis in Denmark: A nationwide cohort study. J Rheumatol 2016; 43: $1335-1339$.

4 Lerang K, Gilboe I, Garen T, Thelle D, Gran J. High incidence and prevalence of systemic lupus erythematosus in Norway. Lupus 2012; 21: $1362-1369$.

5 Hahn BH, McMahon MA, Wilkinson A, et al. American College of Rheumatology guidelines for screening, treatment, and manage- ment of lupus nephritis. Arthritis Care Res (Hoboken) 2012; 64: 797-808.
6 Bertsias GK, Tektonidou M, Amoura Z, et al. Joint European League Against Rheumatism and European Renal AssociationEuropean Dialysis and Transplant Association (EULAR/ERAEDTA) recommendations for the management of adult and paediatric lupus nephritis. Ann Rheum Dis 2012; 71: 1771-1782.

7 Weening JJ, D'Agati VD, Schwartz MM, et al. The Classification of glomerulonephritis in systemic lupus erythematosus revisited. $J$ Am Soc Nephrol 2004; 15: 241-250.

8 Rovin BH, Stillman IE. The Kidney in systemic lupus erythematosus. In: Lahita RG (ed.), Systemic lupus erythematosus, 5th ed. London, UK: Academic Press, 2011. pp. 769-814.

9 Cervera R, Khamashta MA, Hughes GR. The Euro-lupus project: Epidemiology of systemic lupus erythematosus in Europe. Lupus 2009; 18: 869-874.

10 Bono L, Cameron JS, Hicks JA. The very long-term prognosis and complications of lupus nephritis and its treatment. QJM 1999; 92 : 211-218.

11 Cervera R, Khamashta MA, Font J, et al. Morbidity and mortality in systemic lupus erythematosus during a 10-year period: A comparison of early and late manifestations in a cohort of 1,000 patients. Medicine (Baltimore) 2003; 82: 299-308.

12 Faurschou M, Dreyer L, Kamper AL, Starklint H, Jacobsen S. Longterm mortality and renal outcome in a cohort of 100 patients with lupus nephritis. Arthritis Care Res (Hoboken) 2010; 62: 873-880.

13 Danila MI, Pons-Estel GJ, Zhang J, Vila' LM, Reveille JD, Alarco' $\mathrm{n}$ GS. Renal damage is the most important predictor ofmortality within the damage index: Data from LUMINA LXIV, a multiethnic US cohort. Rheumatology (Oxford) 2009; 48: 542-545.

14 Mok CC, Kwok RC, Yip PS. Effect of renal disease on the standardized mortality ratio and life expectancy of patients with systemic lupus erythematosus. Arthritis Rheum 2013; 65: 2154-2160.

15 Gilboe IM, Husby G. Application of the 1982 revised criteria for the classification of systemic lupus erythematosus on a cohort of 346 Norwegian patients with connective tissue disease. Scand $J$ Rheumatol 1999; 28: 81-87.

16 Hochberg MC. Updating the American College of Rheumatology revised criteria for the classification of systemic lupus erythematosus. Arthritis Rheum 1997; 40: 1725.

17 StatBank Norway. Statistics Norway, 2013, https://www.ssb.no/ statbank/ (accessed August 2014).

18 Rothman KJ, Boice JD Jr. Epidemiologic analysis with a programmable calculator. Bethesda, MD: US Department of Health, Education, and Welfare, Public Health Service, National Institutes of Health, 1979.

19 Voss A, Green A, Junker P. Systemic lupus erythematosus in Denmark: Clinical and epidemiological characterization of acountybased cohort. Scand J Rheumatol 1998; 27: 98-105.

20 Lim S, Bayakly AR, Helmick CG, Gordon C, Easley KA, Drenkard $\mathrm{C}$. The incidence and prevalence of systemic lupus ery- thematosus, 2002-2004: The Georgia Lupus Registry. Arthritis Rheumatol 2014; 66: $357-368$.

21 Ingvarsson RF, Bengtsson AA, Jo“ nsen A. Variations in the epidemiology of systemic lupus erythematosus in southern Sweden. Lupus 2016; 25: 772-780.

22 Eilertsen GØ, Fismen S, Hanssen TA, Nossent JC. Decreased incidence of lupus nephritis in northern Norway is linked to increased use of antihypertensive and anticoagulant therapy. Nephrol Dial Transplant 2011; 26: 620-627.

23 Laustrup H, Voss A, Green A, Junker P. SLE disease patterns in a Danish population-based lupus cohort: An 8-year prospective study. Lupus 2010; 19: 239-246.

24 Patel M, Clarke AM, Bruce IN, Symmons DP. The prevalence and incidence of biopsy-proven lupus nephritis in the UK: Evidence of an ethnic gradient. Arthritis Rheum 2006; 54: 2963-2969.

25 Izmirly PM, Wan I, Sahl S, et al. The incidence and prevalence of systemic lupus erythematosus in New York County (Manhattan), New York: The Manhattan Lupus Surveillance Program. Arthritis Rheumatol 2017; 69: 2006-2017.

26 Hanly JG, O'Keeffe AG, Su L, et al. The frequency and outcome of lupus nephritis: Results from an international inception cohort study. Rheumatology (Oxford) 2016; 55: 252-262. 
27 Croca SC, Rodrigues T, Isenberg DA. Assessment of a lupus nephritis cohort over a 30-year period. Rheumatology (Oxford) 2011; 50 1424-1430.

28 Galindo-Izquierdo M, Rodriguez-Almaraz E, Pego-Reigosa JM, et al. Characterization of patients with lupus nephritis included in a large cohort from the Spanish Society of Rheumatology Registry of Patients with Systemic Lupus Erythematosus (RELESSER). Medicine (Baltimore) 2016; 95: e2891.

29 Duarte-Garc1' a A, Barr E, Magder LS, Petri M. Predictors of incident proteinuria among patients with SLE. Lupus Sci Med 2017; 4: e000200.

30 Tanha N, Hansen RB, Nielsen CT, Faurschou M, Jacobsen S. Clinical and serological associations with the development of incident proteinuria in Danish patients with systemic lupus erythematosus. J Rheumatol 2018; 45: 934-941.

31 Bastian HM, Roseman JM, McGwin G Jr, et al. Systemic lupus erythematosus in three ethnic groups. XII. Risk factors for lupus nephritis after diagnosis. Lupus 2002; 11: 152-160.

32 Pons-Estel GJ, Aspey LD, Bao G, et al. Early discoid lupus erythematosus protects against renal disease in patients with systemic lupus erythematosus: Longitudinal data from a large Latin American cohort. Lupus 2017; 26: 73-83.

33 Mosca M, Tani C, Aringer M, et al. European League Against Rheumatism recommendations for monitoring patients with systemic lupus erythematosus in clinical practice and in observational studies. Ann Rheum Dis 2010; 69: 1269-1274.

34 Health expenditure per capita The World Bank, 2014, https://databank.worldbank.org/data/ reports.aspx ? source $=2 \&$ series $=$ SH.XPD.CHEX.GD.ZS\&countr (accessed September 2017).

35 Plantinga L, Lim SS, Patzer R, et al. Incidence of end-stage renal disease among newly diagnosed systemic lupus erythematosus patients: The Georgia Lupus Registry. Arthritis Care Res (Hoboken) 2016; 68: 357-365.

$36 \mathrm{Yu} \mathrm{KH,} \mathrm{Kuo} \mathrm{CF,} \mathrm{Chou} \mathrm{IJ,} \mathrm{Chiou} \mathrm{MJ,} \mathrm{See} \mathrm{LC.} \mathrm{Risk} \mathrm{of} \mathrm{end-stage}$ renal disease in systemic lupus erythematosus patients: A nationwide population-based study. Int J Rheum Dis 2016; 19: 1175-1182.

37 Gagnum V, Saeed M, Stene LC, Leivestad T, Joner G, Skrivarhaug T. Low incidence of end-stage renal disease in child- hood-onset type 1 diabetes followed for up to 42 years. Diabetes Care 2018; 41 : $420-425$.

38 Voss A, Laustrup H, Hjelmborg J, Junker P. Survival in systemic lupus erythematosus, 1995-2010. A prospective study in a Danish community. Lupus 2013; 22: 1185-1191.

39 Lerang K, Gilboe IM, Steinar Thelle D, Gran JT. Mortality and years of potential life loss in systemic lupus erythematosus: A populationbased cohort study. Lupus 2014; 23: 1546-1552.

40 Yurkovich M, Vostretsova K, Chen W, Avin a-Zubieta JA. Overall and cause-specific mortality in patients with systemic lupus erythematosus: A meta-analysis of observational studies. Arthritis Care Res (Hoboken) 2014; 66: 608-616.

41 Lee YH, Choi SJ, Ji JD, Song GG. Overall and cause-specific mortality in systemic lupus erythematosus: An updated meta-analysis. Lupus 2016; 25: 727-734.

42 Norby GE, Mjøen G, Bjørneklett R, et al. Outcome in biopsyproven lupus nephritis: Evaluation of biopsies from the Norwegian Kidney Biopsy Registry. Lupus 2017; 26: 881-885.

43 Kono M, Yasuda S, Kato M, et al. Long-term outcome in Japanese patients with lupus nephritis. Lupus 2014; 23:1124-1132.

44 Yap DY, Tang CS, Ma MK, Lam MF, Chan TM. Survival ana- 1ysis and causes of mortality in patients with lupus nephritis. Nephrol Dial Transplant 2012; 27: 3248-3254.

45 Bernatsky S, Boivin JF, Joseph L, et al. Mortality in systemic lupus erythematosus. Arthritis Rheum 2006; 54: 2550-2557. 